\title{
Beam Energy Dependence of Hanbury-Brown-Twiss Radii from a Blast-Wave Model
}

\author{
S. Zhang, ${ }^{1}$ Y. G. Ma, ${ }^{1,2}$ J. H. Chen, ${ }^{1}$ and C. Zhong ${ }^{1}$ \\ ${ }^{1}$ Shanghai Institute of Applied Physics, Chinese Academy of Sciences, Shanghai 201800, China \\ ${ }^{2}$ ShanghaiTech University, Shanghai 200031, China
}

Correspondence should be addressed to Y. G. Ma; ygma@sinap.ac.cn

Received 25 May 2016; Revised 9 September 2016; Accepted 9 October 2016

Academic Editor: Ming Liu

Copyright ( $(2016$ S. Zhang et al. This is an open access article distributed under the Creative Commons Attribution License, which permits unrestricted use, distribution, and reproduction in any medium, provided the original work is properly cited. The publication of this article was funded by SCOAP ${ }^{3}$.

\begin{abstract}
The beam energy dependence of correlation lengths (the Hanbury-Brown-Twiss radii) is calculated by using a blast-wave model and the results are comparable with those from RHIC-STAR beam energy scan data as well as the LHC-ALICE measurements. A set of parameters for the blast-wave model as a function of beam energy under study are obtained by fit to the HBT radii at each energy point. The transverse momentum dependence of $\mathrm{HBT}$ radii is presented with the extracted parameters for $\mathrm{Au}+\mathrm{Au}$ collision at $\sqrt{s_{\mathrm{NN}}}=200 \mathrm{GeV}$ and for $\mathrm{Pb}+\mathrm{Pb}$ collisions at $2.76 \mathrm{TeV}$. From our study one can learn that particle emission duration cannot be ignored while calculating the HBT radii with the same parameters. And tuning kinetic freeze-out temperature in a range will result in system lifetime changing in the reverse direction as it is found in RHIC-STAR experiment measurements.
\end{abstract}

\section{Introduction}

The Quark-Gluon-Plasma (QGP) predicted by quantum chromodynamics (QCD) [1] can be formed in relativistic heavy-ion collisions. It is believed that this kind of new state of matter is produced in the early stage of central $\mathrm{Au}+\mathrm{Au}$ collisions at the top energy in the Relativistic Heavy-Ion Collider (RHIC) at Brookhaven National Laboratory [2-5]. It was concluded that the hot-dense matter is a strongly interacting partonic matter named as sQGP under extreme temperature and energy density with sufficient experimental evidences [6-10]. Recently, many results in $\mathrm{Pb}+\mathrm{Pb}$ and $\mathrm{p}+\mathrm{Pb}$ collisions at $\sqrt{s_{\mathrm{NN}}}=2.76 \mathrm{TeV}$ in the Large Hadron Collider (LHC) were also reported for exploring properties of the hotdense quark-gluon matter [11-14].

Mapping the QCD phase diagram and locating the phase boundary and possible critical end point become hot topic in the field $[1,15-18]$. The properties inherited from QGP will imprint signal on observables which can reflect phase transition information. The geometry of the system shall undergo phase space evolution from QGP stage to hadron kinetic freeze-out stage, which can be considered as an observable that is sensitive to the equation of state $[19,20]$. Hanbury-Brown-Twiss (HBT) technique invented for measuring sizes of nearby stars [21] was extended to particle physics [22] and heavy-ion collisions [23-31]. The HBT technique can also be applied to extract the precise space-time properties from particle emission region at kinetic freeze-out stage in heavy-ion collisions. Furthermore this technique has been evolved to search for new particles and to measure particle interactions [32-34].

Experimental results on HBT study in high energy nuclear reaction were reported by STAR $[35,36]$ and PHENIX $[37,38]$ at RHIC top energy in $\mathrm{Au}+\mathrm{Au}$ collisions, as well as by ALICE [39] at $\sqrt{s_{\mathrm{NN}}}=2.76 \mathrm{TeV}$ in $\mathrm{Pb}+\mathrm{Pb}$ collisions. Recently STAR and PHENIX collaborations have also presented beam energy dependence of HBT radii $[20,40]$ and a nonmonotonic changing behaviour for the square difference between outward radius and sideward radius $\left(R_{\text {out }}^{2}-R_{\text {side }}^{2}\right)$ with increase of beam energy was found. This behaviour could be sensitive to equation of state and was considered as a probe related to the critical end point of QGP phase transition [19]. A finite-size scaling (FSS) analysis of experimental data was performed in [19] and the analysis suggested that a second-order phase transition took place with a critical end point located at a chemical freeze-out temperature of $\sim 165 \mathrm{MeV}$ and a baryon chemical potential of $\sim 95 \mathrm{MeV}$. 
In this paper we present beam energy dependence of HBT radii calculated from a blast-wave model. Firstly, experimental data of HBT radii from RHIC-STAR and LHC-ALICE are fitted and parameters for the blast-wave model are configured as a function of beam energy. The transverse momentum dependence of HBT radii is calculated at RHIC top energy and LHC energy with these parameters. From the results, it was found that particle emission duration is important for calculating transverse momentum dependence of $\mathrm{HBT}$ radii and changing of kinetic freeze-out temperature will result in system lifetime changing in reverse direction as that in the RHIC-STAR experimental analysis [20].

The paper is organised as follows. In Section 2, blast-wave model and HBT correlation function are briefly introduced. Some kinetic parameters are presented as a function of beam energy. Section 3 presents energy dependence of extracted HBT radii with various kinetic temperatures, system lifetime, particle emission duration, and so forth. Transverse momentum dependence of HBT radii is discussed in Section 4. Finally Section 5 gives the summary.

\section{Blast-Wave Model and HBT Correlation Function}

The particle emission function $S(x, p)$ in heavy-ion collisions used in this study is similar as in [44]

$$
\begin{aligned}
& S(x, p)=m_{T} \cosh (\eta-Y) \Omega\left(r, \phi_{s}\right) e^{-\left(\tau-\tau_{0}\right)^{2} / 2 \Delta \tau^{2}} \\
& \cdot \frac{1}{e^{K \cdot u / T_{\text {kin }}} \pm 1}=m_{T} \cosh (\eta-Y) \Omega\left(r, \phi_{s}\right) \\
& \cdot e^{-\left(\tau-\tau_{0}\right)^{2} / 2 \Delta \tau^{2}} \sum_{n=1}^{\infty}(\mp)^{n+1} e^{-K \cdot u / T_{\text {kin }}} \simeq m_{T} \cosh (\eta-Y) \\
& \cdot \Omega\left(r, \phi_{s}\right) e^{-\left(\tau-\tau_{0}\right)^{2} / 2 \Delta \tau^{2}} e^{-K \cdot u / T_{\text {kin }}} .
\end{aligned}
$$

In cylindrical coordinates, source moving four-velocity and momentum can be written, respectively, as

$$
\begin{gathered}
u_{\mu}(x)=\left(\cosh \eta \cosh \rho\left(r, \phi_{s}\right), \sinh \rho\left(r, \phi_{s}\right)\right. \\
\left.\cdot \cos \phi_{b}, \sinh \rho\left(r, \phi_{s}\right) \sin \phi_{b}, \sinh \eta \cosh \phi_{b}\right), \\
K_{\mu}=\left(m_{T} \cosh Y, p_{T} \cos \phi_{p}, p_{T} \sin \phi_{p}, m_{T} \sinh Y\right) .
\end{gathered}
$$

And the flow rapidity is given by

$$
\rho\left(r, \phi_{s}\right)=\tilde{r}\left[\rho_{0}+\rho_{2} \cos \left(2 \phi_{b}\right)\right]
$$

here the normalized elliptical radius is as follows:

$$
\widetilde{r} \equiv \sqrt{\frac{\left[r \cos \phi_{s}\right]^{2}}{R_{x}^{2}}+\frac{\left[r \sin \phi_{s}\right]^{2}}{R_{y}^{2}}},
$$

with

$$
\tan \phi_{s}=\left(\frac{R_{y}}{R_{x}}\right)^{2} \tan \phi_{b} .
$$

In (1), spatial weighting of source elements is selected as a simple pattern [44]:

$$
\Omega\left(r, \phi_{s}\right)= \begin{cases}1, & \tilde{r}<1 \\ 0, & \tilde{r}>1 .\end{cases}
$$

Here are the main parameters in this model, the kinetic freeze-out temperature $T_{\text {kin }}$, the radial flow parameter $\rho_{0}$, the "elliptic flow parameter" $\rho_{2}$ which controls second-order oscillation of transverse rapidity by the relation as in (5), the system lifetime $\tau_{0}$, and the particle emission durations $\Delta \tau, R_{x}$, and $R_{y}$ related to system size and space asymmetry. In this calculation we assume that the system is in most central heavy-ion collisions and thus set the $R_{x}=R_{y}=R_{0}$, $\rho_{2}=0$. In experimental measurement, hadron spectra can be fitted by the blast-wave model with integrating the emission function except $p_{T}$ and $Y$. The kinetic freeze-out temperature $T_{\text {kin }}$ and the averaged radial flow $\langle\beta\rangle$ were extracted from the fit. For detailed technique information, one may refer to [41]. The averaged radial flow is related to the flow rapidity $\rho=\tanh ^{-1} \beta$, from which the radial flow parameter $\rho_{0}$ is calculated. Figures 1 and 2 present the measured $T_{\text {kin }}$ and $\langle\beta\rangle$ at a wide beam energy range, respectively. The data come from $[41,42]$. The kinetic freeze-out temperature $T_{\text {kin }}$ and the averaged radial flow $\langle\beta\rangle$ can be parametrised as a function of $\sqrt{s_{\mathrm{NN}}}$ by empirical formula:

$$
\begin{aligned}
T_{\text {kin }} & =T_{\lim } \\
\cdot & \frac{1}{\left(1+\exp \left(8.559-\ln \left(\sqrt{s_{\mathrm{NN}}}\right) / 0.093\right)\right) /\left({\sqrt{s_{\mathrm{NN}}}}^{0.057} / 0.846\right)}, \\
\langle\beta\rangle & =\beta_{\lim } \frac{1}{\left(1+\exp \left(5.666-\ln \left(\sqrt{s_{\mathrm{NN}}}\right) / 0.124\right)\right){\sqrt{s_{\mathrm{NN}}}}^{0.065}},
\end{aligned}
$$

where $T_{\lim }=169.171 \mathrm{MeV}$ and $\beta_{\lim }=0.399$. And then free parameters in the blast-wave model will be $R_{0}, \tau_{0}$, and $\Delta \tau$, which are all related to expanding characters of the collision system. And it will be determined by the HBT correlation calculation which will be discussed below in detail.

In our previous works, the blast-wave model was coupled with thermal equilibrium model to describe the hadron production and its spectra with a range of thermal parameters [45] and with coalescence mechanism to calculate the light nuclei production and to predict the di-baryons production rate $[46,47]$. In addition, the DRAGON model [48] and the THERMINATOR2 $[49,50]$ model have also been developed as event generator to study the phase space distribution of hadrons at freeze-out stage. It is also successfully applied in experimental data analysis $[41,42]$ to extract the kinetic freeze-out properties and to provide the phase space distribution to calculate the HBT correlation in theory $[44,51]$.

The identical two particle HBT correlation function can be written as $[26,51]$

$$
C(\vec{K}, \vec{q})=1+\left|\frac{\int d^{4} x e^{i \vec{q} \cdot(\vec{x}-\vec{\beta} t)} S(x, K)}{\int d^{4} x S(x, K)}\right|,
$$

here $K$ is average momentum for the two particles, $K=$ $(1 / 2)\left(p_{1}+p_{2}\right), q$ denotes relative momentum between two 


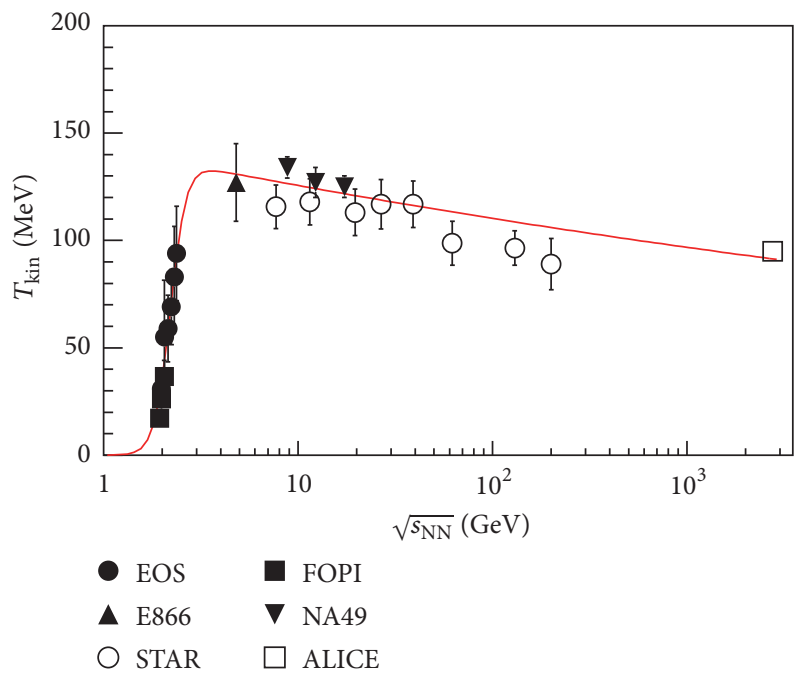

FIgURE 1: Kinetic freeze-out temperature as a function of centre-ofmass energy $\sqrt{s_{\mathrm{NN}}}$. Data are from $[41,42]$.

particles, $q=p_{1}-p_{2}$, and $\vec{\beta}=\vec{K} / \vec{K}_{0}$. From [44, 51-53], the "out-side-long" coordinates system is used in this calculation, in which the long direction $R_{\text {long }}$ is parallel to the beam, the sideward direction $R_{\text {side }}$ is perpendicular to the beam and total pair momentum, and the outward direction $R_{\text {out }}$ is perpendicular to the long and sideward directions. After expanding angular dependence of $C(K, q)$ in a harmonic series with the "out-side-long" coordinates system, the HBT radii can be written as $[44,51]$

$$
\begin{aligned}
R_{\text {side }}^{2}= & \frac{1}{2}\left(\left\langle\tilde{x}^{2}\right\rangle+\left\langle\tilde{y}^{2}\right\rangle\right) \\
& -\frac{1}{2}\left(\left\langle\tilde{x}^{2}\right\rangle-\left\langle\tilde{y}^{2}\right\rangle\right) \cos \left(2 \phi_{p}\right) \\
& -\langle\tilde{x} \tilde{y}\rangle \sin \left(2 \phi_{p}\right), \\
R_{\text {out }}^{2}= & \frac{1}{2}\left(\left\langle\tilde{x}^{2}\right\rangle+\left\langle\tilde{y}^{2}\right\rangle\right) \\
& +\frac{1}{2}\left(\left\langle\tilde{x}^{2}\right\rangle-\left\langle\tilde{y}^{2}\right\rangle\right) \cos \left(2 \phi_{p}\right) \\
& +\langle\tilde{x} \tilde{y}\rangle \sin \left(2 \phi_{p}\right) \\
& -2 \beta_{T}\left(\langle\tilde{t} \tilde{x}\rangle \cos \phi_{p}+\langle\tilde{t} \tilde{y}\rangle \sin \phi_{p}\right) \\
& +\beta_{T}^{2}\left\langle\tilde{t}^{2}\right\rangle, \\
R_{\text {long }}^{2}= & \left\langle\widetilde{z}^{2}\right\rangle-2 \beta_{l}\langle\tilde{t} \tilde{z}\rangle+\beta_{l}^{2}\left\langle\tilde{t}^{2}\right\rangle,
\end{aligned}
$$

where

$$
\begin{aligned}
\langle f(x)\rangle(K) & \equiv \frac{\int d^{4} x f(x) S(x, K)}{\int d^{4} x S(x, K)}, \\
\widetilde{x}^{\mu} & =x^{\mu}-\left\langle\widetilde{x}^{\mu}\right\rangle(K) .
\end{aligned}
$$

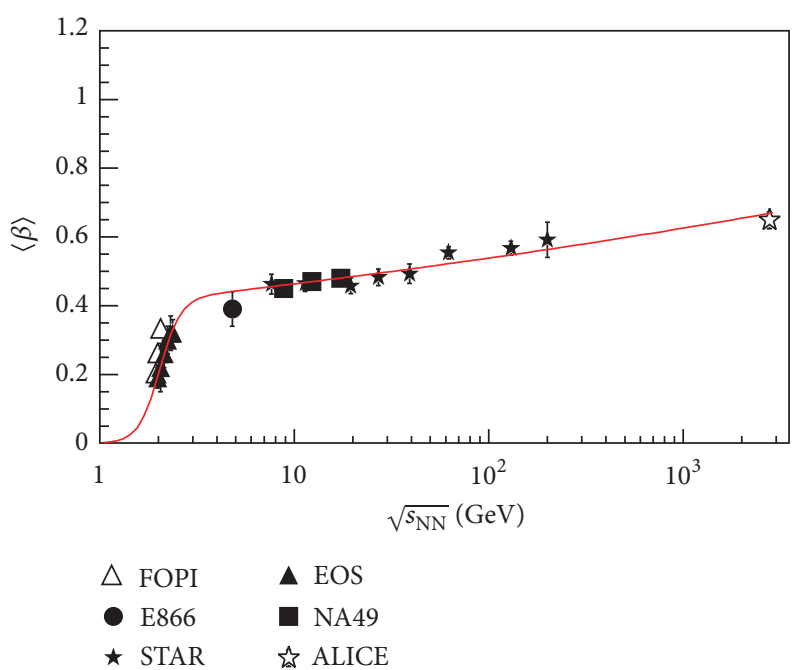

FIgURE 2: The averaged radial flow $\langle\beta\rangle$ as a function of centre-ofmass energy $\sqrt{s_{\mathrm{NN}}}$. Data are from $[41,42]$.

In the calculation, observables are related to integrals of emission function (1) over phase space $d^{4} x=d x d y d z d t=$ $\tau d \tau d \eta r d r d \phi_{s}$, weighted with some quantities $B(x, K)$. If $B(x, K)=B^{\prime}\left(r, \phi_{s}, K\right) \tau^{i} \sinh ^{j} \eta \cosh ^{k} \eta$, then the integrals can be written as in [44]

$$
\begin{aligned}
& \int_{0}^{2 \pi} d \phi_{s} \int_{0}^{\infty} r d r \int_{-\infty}^{\infty} d \eta \int_{-\infty}^{\infty} \tau d \tau S(x, K) B(x K) \\
& \quad=m_{T} H_{i}\left\{B^{\prime}\right\}_{j, k}(K)
\end{aligned}
$$

and some useful integrals

$$
\begin{aligned}
& H_{i} \equiv \int_{-\infty}^{\infty} d \tau \tau^{i+1} e^{-\left(\tau-\tau_{0}\right)^{2} / 2 \Delta \tau^{2}}, \\
& G_{j, k}(x, K) \equiv \int_{-\infty}^{\infty} d \eta e-\beta \cosh \eta \sinh ^{j} \eta \cosh ^{k+1} \eta, \\
& \left\{B^{\prime}\right\}_{j, k}(K) \equiv \int_{0}^{2 \pi} d \phi_{s} \int_{0}^{\infty} r d r G_{j, K}(x, K) B^{\prime}(x, K) \\
& \cdot e^{\alpha \cos \left(\phi_{b}-\phi_{p}\right)} \Omega\left(r, \phi_{s}\right),
\end{aligned}
$$

where we define

$$
\begin{aligned}
& \alpha \equiv \frac{p_{T}}{T} \sinh \rho\left(r, \phi_{s}\right), \\
& \beta \equiv \frac{m_{T}}{T} \cosh \rho\left(r, \phi_{s}\right) .
\end{aligned}
$$

Retière and Lisa [44] have provided a systematic analysis of parameter range for the blast-wave model and investigated the $p_{T}$ spectra, the collective flow, and the HBT correlation of hadrons produced in heavy-ion collisions. In this calculation we will use the algorithm developed in $[44,51]$ to study the energy and transverse momentum dependence of pion HBT correlation radii. Based on the discussion above, the free parameters will be $R_{0}, \tau_{0}$, and $\Delta \tau$ which can be determined 


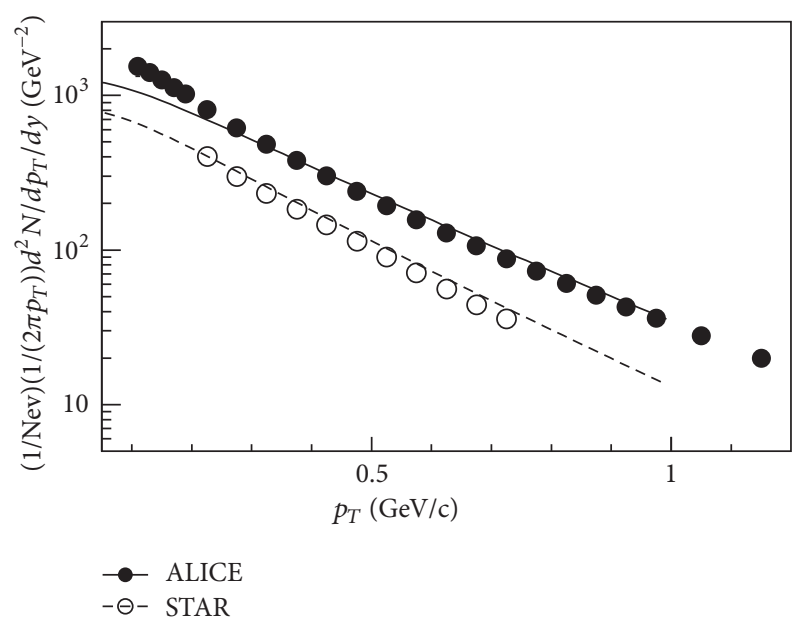

Figure 3: Comparison of pion's spectra from blast-wave model (lines) and the data in central Au+Au collisions at $\sqrt{s_{\mathrm{NN}}}=200 \mathrm{GeV}$ [43] and the data in central $\mathrm{Pb}+\mathrm{Pb}$ collisions at $\sqrt{s_{\mathrm{NN}}}=2.76 \mathrm{TeV}$ [14].

by fitting experimental data by (9). Before the study of energy dependence on HBT radii, we calculated pion's spectra by using this algorithm in the blast-wave model:

$$
\frac{d N}{p_{T} d p_{T}}=\int d \phi_{p} \int d^{4} x S(x, K)
$$

Figure 3 presents pion's spectra which are comparable with experimental data from STAR at $\sqrt{s_{\mathrm{NN}}}=200 \mathrm{GeV}$ in central $\mathrm{Au}+\mathrm{Au}$ collisions [43] and ALICE at $\sqrt{s_{\mathrm{NN}}}=2.76 \mathrm{TeV}$ in central $\mathrm{Pb}+\mathrm{Pb}$ collisions [14], respectively.

\section{Energy Dependence of HBT Radii}

The parameters are configured as following. The kinetic freeze-out temperature $T_{\text {kin }}$ and the averaged radial flow $\langle\beta\rangle$ are from (7) as a function of $\sqrt{s_{\mathrm{NN}}}$, but in some cases $T_{\text {kin }}$ are fixed to 90,100 and $120 \mathrm{MeV}$ for comparison. In numerical calculation, the particle emission duration $\Delta \tau$ is set to zero and in another case the energy dependence of $\Delta \tau$ will be extracted by fit on the data. The $R_{0}$ will also be given by fit the data at each energy point. The experimental results of HBT radii are taken from the STAR and the ALICE collaborations $[20,39]$ at centre-of-mass energy $\sqrt{s_{\mathrm{NN}}}$ points, 7.7, 11.5, 19.6, 27, 39, 62.4, 200, and $2760 \mathrm{GeV}$. The difference between calculated radii results and the experimental data should reach a minimum value $\left(\delta_{s}, \delta_{o}, \delta_{l}\right)$ for each energy point:

$$
\begin{gathered}
R_{\text {side }}(\text { th })-R_{\text {side }}(\exp )=\delta_{s}, \\
R_{\text {out }}(\text { th })-R_{\text {out }}(\exp )=\delta_{o}, \\
R_{\text {long }}(\text { th })-R_{\text {long }}(\exp )=\delta_{l} .
\end{gathered}
$$

Actually from (9) and the algorithm in [44, 51], one can find the HBT radii parameter dependence as follows:

$$
\begin{aligned}
& R_{\text {side }}^{2}=R_{\text {side }}^{2}\left(T_{\text {kin }}, \rho_{0}, R_{0}\right), \\
& R_{\text {out }}^{2}=R_{\text {out }}^{2}\left(T_{\text {kin }}, \rho_{0}, R_{0}, \tau_{0}, \Delta \tau\right), \\
& R_{\text {long }}^{2}=R_{\text {long }}^{2}\left(T_{\text {kin }}, \rho_{0}, R_{0}, \tau_{0}, \Delta \tau\right) .
\end{aligned}
$$

So $R_{0}$ can be determined directly by fit on $R_{\text {side }}^{2}$. And $\tau_{0}$ and $\Delta \tau$ can be extract by fit on $R_{\text {out }}^{2}$ and $R_{\text {long }}^{2}$ simultaneously. We then learnt that the difference of $R_{\text {out }}^{2}-R_{\text {side }}^{2}$ depends not only on the system lifetime $\tau_{0}$ but also on the particle emission duration $\Delta \tau$.

Figure 4 presents our calculation on HBT radii for identical charged pion-pion correlation with the configured parameters. The HBT radii show an increasing trend with the increasing of centre-of-mass energy $\sqrt{s_{\mathrm{NN}}}$. In the case of $\Delta \tau \neq$ 0 , the results can describe experimental data successfully. However, for $\Delta \tau=0.0, R_{\text {out }}$ cannot be fitted despite the fact that $R_{\text {long }}$ can be well matched by the calculation. Since $T_{\text {kin }}$ and $\rho_{0}$ are taken from experimental results, $R_{\text {side }}$ will only depend on parameter $R_{0}$, which reflects the system size where particles are emitted. Figure 5 displays the extracted $R_{0}$ as a function of $\sqrt{s_{\mathrm{NN}}}$. It demonstrates a similar trend of energy dependence as $R_{\text {side }}$. With fixed temperature of $T_{\text {kin }}(90,100$, and $120 \mathrm{MeV}$ ), it is found that a large $R_{0}$ is needed to fit the data while $T_{\text {kin }}$ sets to small value. This is consistent with evolution of the fireball created in heavy-ion collisions, where temperature becomes lower while system size increases.

$R_{\text {out }}$ and $R_{\text {long }}$ depend not only on $\tau_{0}$ but also on $\Delta \tau$. Figure 6 shows $\tau_{0}$ and $\Delta \tau$ as a function of centre-of-mass energy $\sqrt{s_{\mathrm{NN}}}$ from fit to the data. $\Delta \tau$ slightly depends on the $\sqrt{s_{\mathrm{NN}}}$. From Figure 6 one can see that $\tau_{0}$ generally increases with the increasing of $\sqrt{s_{\mathrm{NN}}}$ in trends but there exists a minimum value at $\sqrt{s_{\mathrm{NN}}} \sim 39 \mathrm{GeV}$. It may imply that the system in higher energy (such as at LHC) will undergo a longer time evolution than in lower energy before hadron rescattering ceases (the kinetic freeze-out status). With fixed temperature of $T_{\text {kin }}(90,100$, and $120 \mathrm{MeV})$, the system lifetime $\tau_{0}$ and the particle emission duration $\Delta \tau$ are all in reverse order to the temperature $T_{\text {kin }}$. This suggests that a system expanding with a long lifetime and a broad duration will result in a lower temperature, which is consistent with the behaviour of $R_{0}$ as discussed above. We learnt that our results are comparable with the experimental results with $\Delta \tau \neq 0$. With the system lifetime and HBT radii calculation all taken into account, it can be concluded that the particle emission duration cannot be ignored while fitting the HBT radii $\left(R_{\text {side }}\right.$, $R_{\text {out }}$, and $R_{\text {long }}$ ) at the same time.

After $R_{\text {out }}$ and $R_{\text {side }}$ are all calculated, difference of $R_{\text {out }}^{2}-$ $R_{\text {side }}^{2}$ as a function of centre-of-mass energy $\sqrt{s_{\mathrm{NN}}}$ can be obtained as shown in Figure 7. In the case of $\Delta \tau \neq 0$, the calculated results can describe the data very well. However, it is unsuccessful to fit the data with $\Delta \tau=0$ for the current parameter configuration. Energy dependence of the difference of $R_{\text {out }}^{2}-R_{\text {side }}^{2}$ demonstrates a nonmonotonic 

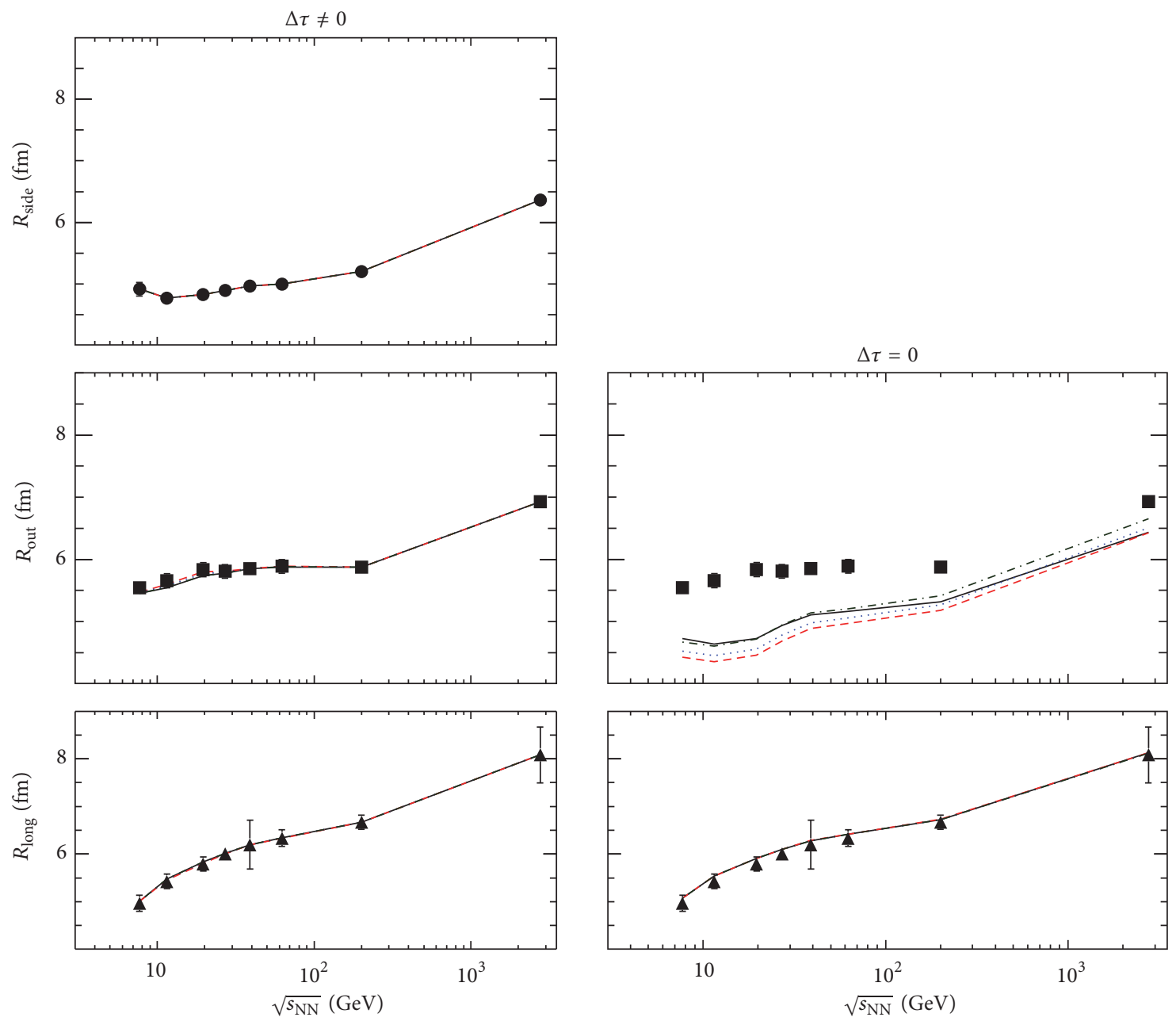

FIGURE 4: The extracted values of $R_{\text {side }}, R_{\text {out }}$, and $R_{\text {long }}$ as a function of centre-of-mass energy $\sqrt{s_{\mathrm{NN}}}$. Different types of lines represent different kinetic temperature parametrisation. Markers are experimental data from [20,39]. Left panels are results with finite particle emission duration $(\Delta \tau)$ and right panels for the cases of $\Delta \tau=0$.

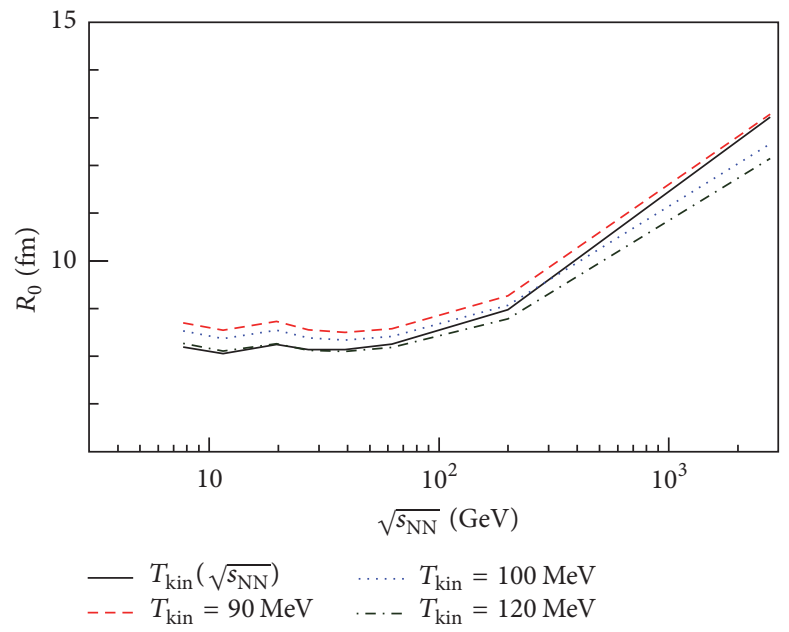

FIGURE 5: $R_{0}$ as a function of centre-of-mass energy $\sqrt{s_{\mathrm{NN}}}$ with different kinetic temperature parametrisation. 

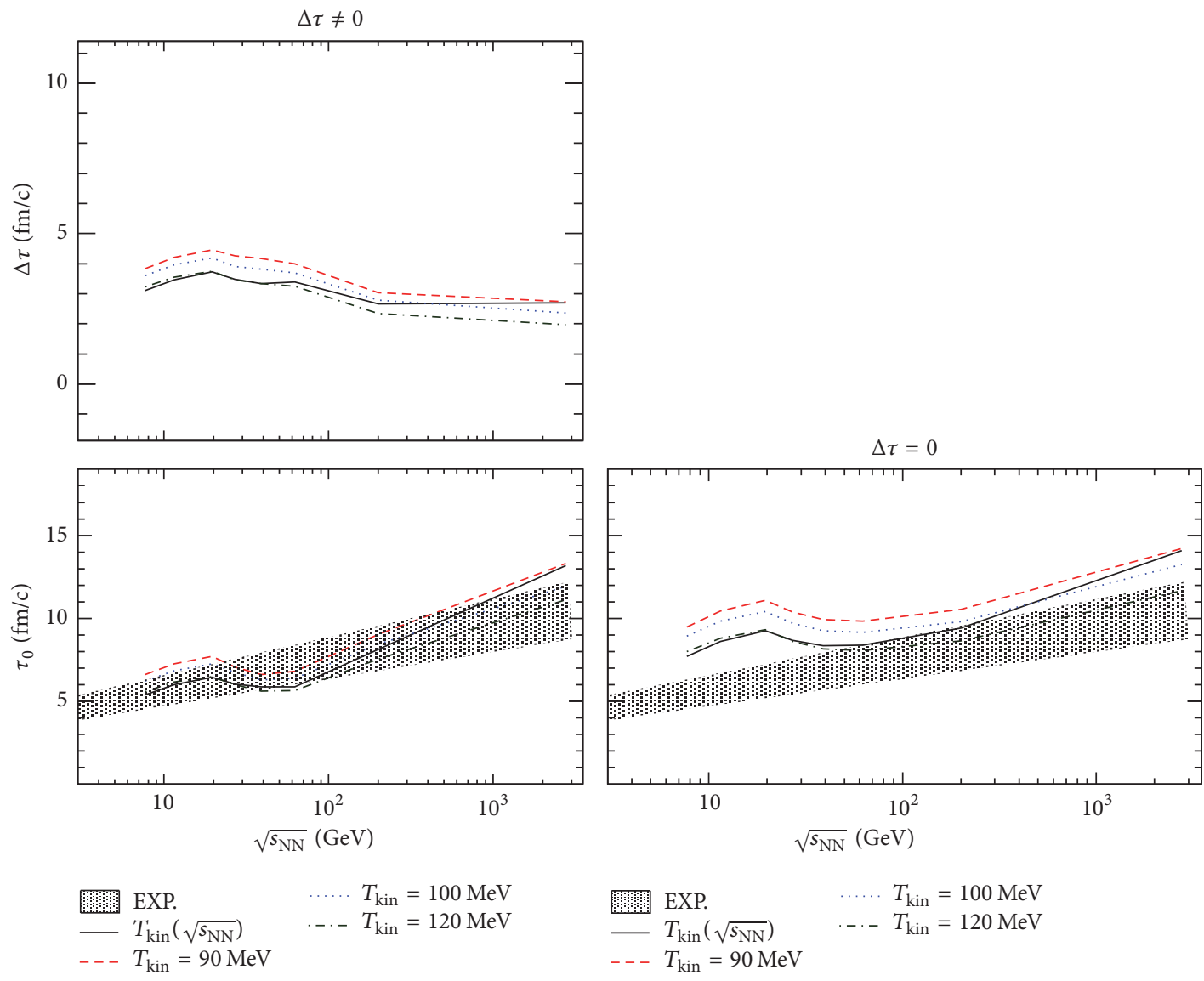

Figure 6: The same as Figure 4 but for $\tau_{0}$ and $\Delta \tau$. Experimental data (shadowed area) is taken from [20].
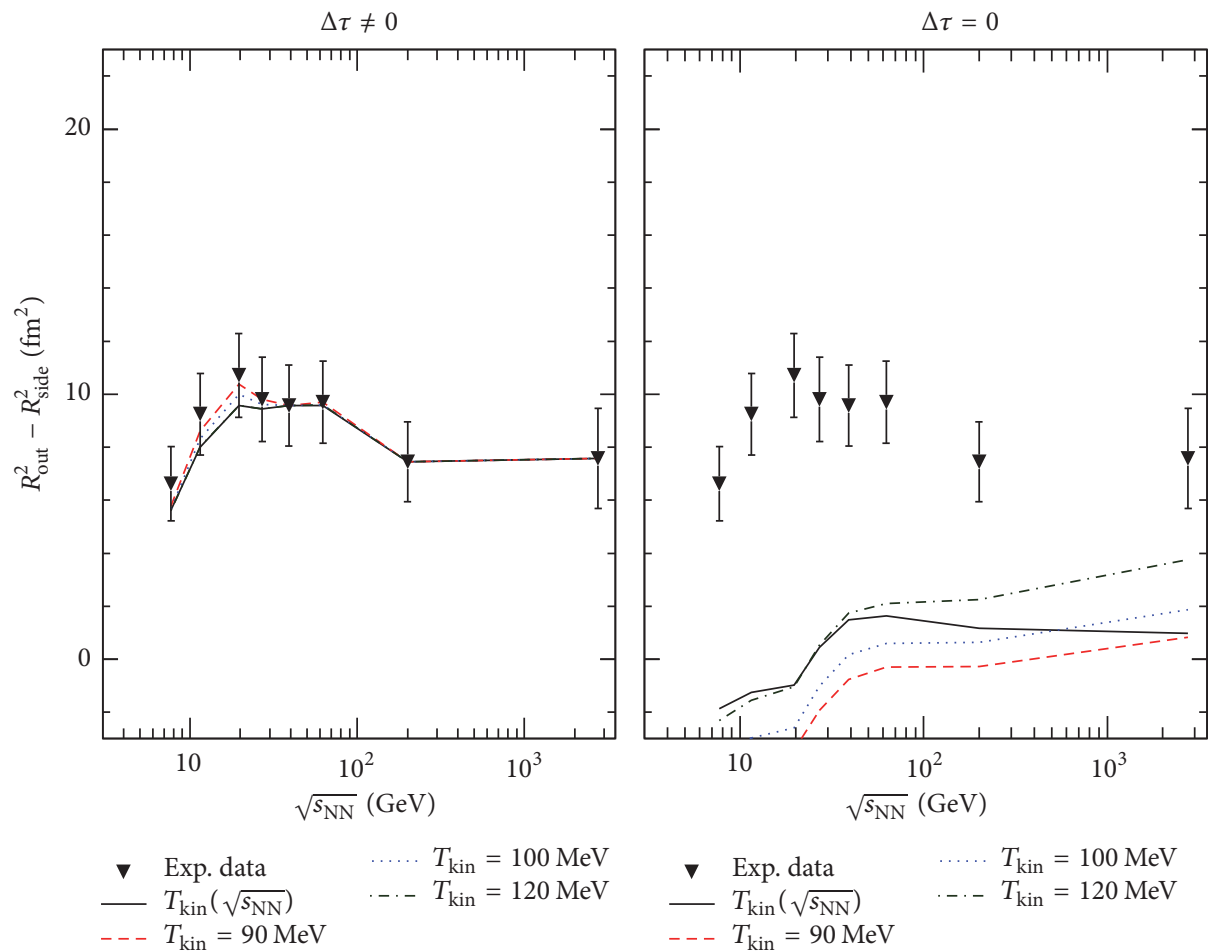

Figure 7: The same as Figure 4 but for the $R_{\text {out }}^{2}-R_{\text {side }}^{2}$. Experiment data is taken from [20]. 

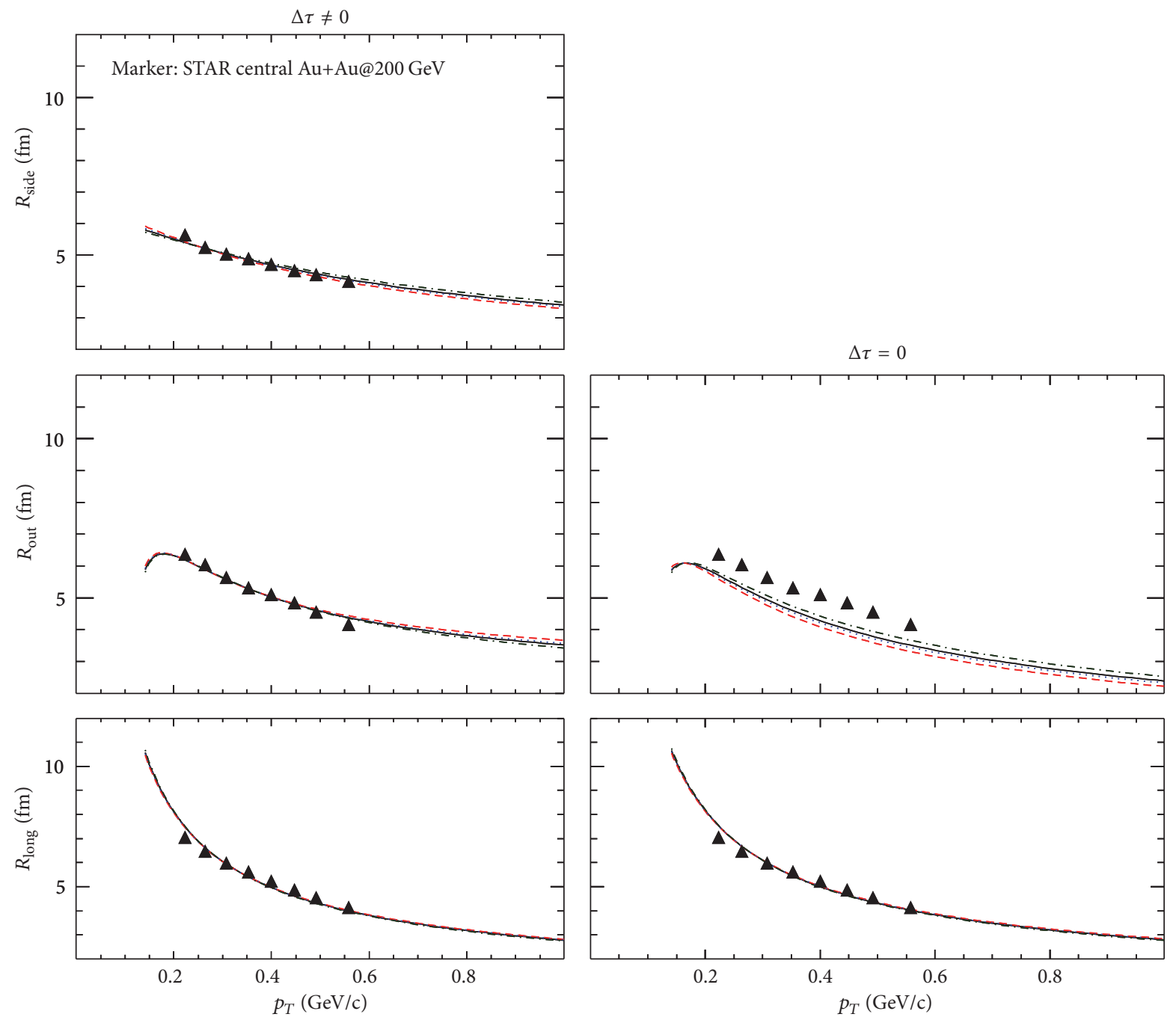

FIgURE 8: The transverse momentum dependence of HBT radii in central Au+Au collisions at $\sqrt{s_{\mathrm{NN}}}=200 \mathrm{GeV}$. Markers are experimental data from [20].

increasing trend with the increasing of $\sqrt{s_{\mathrm{NN}}}$. The peak of experimental results locates at $\sqrt{s_{\mathrm{NN}}} \sim 17.3 \mathrm{GeV}$ [20] and the calculated results give a very similar behaviour for the peak emerging. And in [19], the theoretical work proposes the critical end point (CEP) for deconfinement phase transition at $\sqrt{s_{\mathrm{NN}}}=47.5 \mathrm{GeV}$ by applying FSS. Anyway other observables, such as elliptic flow and fluctuations, should be considered together and other basic theoretical calculations are awaiting for comparison, which contribute to locate the CEP and understand underlying physics around this energy region.

\section{Transverse Momentum Dependence of HBT Radii}

With the above parameter configuration, we also calculated the transverse momentum dependence of $\mathrm{HBT}$ radii at $\sqrt{s_{\mathrm{NN}}}=200 \mathrm{GeV}$ and $2760 \mathrm{GeV}$ in central heavy-ion collisions. Figures 8 and 9 show the HBT radii as a function of transverse momentum in central Au+Au collisions at $\sqrt{s_{\mathrm{NN}}}=$ $200 \mathrm{GeV}$ and in central $\mathrm{Pb}+\mathrm{Pb}$ collisions at $\sqrt{s_{\mathrm{NN}}}=2760 \mathrm{GeV}$, respectively. The experimental data is from $[20,39] . R_{\text {side }}$, $R_{\text {out }}$, and $R_{\text {long }}$ decrease with the increasing of transverse momentum $p_{T}$ as shown in Figure 8, which indicates that high $p_{T}$ particles are emitted from near the centre of the fireball. It is found that the calculated results fit the STAR data in the case of $\Delta \tau \neq 0$ but fails to describe the $R_{\text {out }}$ with $\Delta \tau=0$. The similar $p_{T}$ dependence trend is found in central $\mathrm{Pb}+\mathrm{Pb}$ collisions at $\sqrt{s_{\mathrm{NN}}}=2760 \mathrm{GeV}$ as shown in Figure 9. In the $\Delta \tau \neq 0$ case, the calculated results reproduce the $R_{\text {side }}$ and $R_{\text {out }}$ exactly but slightly underestimate the value of $R_{\text {long }}$. Again, a reasonable parameter configuration cannot be found for fitting ALICE data in the case of $\Delta \tau=0$. These results suggest that the system lifetime and particle emission duration should be taken into account at the same time while describing $R_{\text {side, }}$, $R_{\text {out }}$ and $R_{\text {long }}$ with the same parameter configuration in the blast-wave model. 

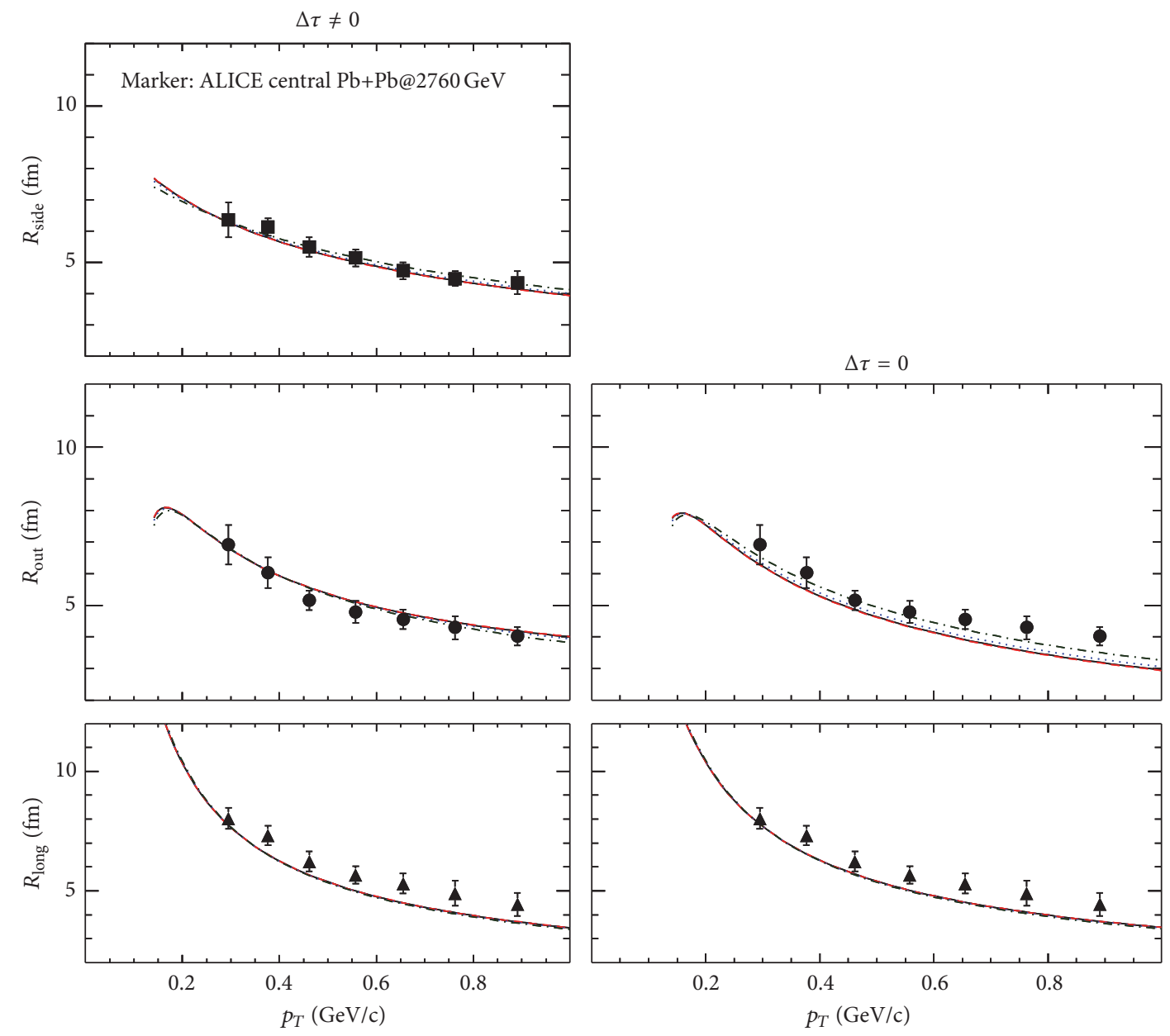

FIgURE 9: The same as Figure 8 but for the central $\mathrm{Pb}+\mathrm{Pb}$ collisions at $\sqrt{\mathcal{S}_{\mathrm{NN}}}=2760 \mathrm{GeV}$. Markers are experimental data from [39].

\section{Summary}

The HBT radii $\left(R_{\text {side }}, R_{\text {out }}\right.$, and $\left.R_{\text {long }}\right)$ are calculated from the blast-wave model in the "out-side-long" (osl) coordinates system. In comparison with the experimental data $[20,39]$, we found that, in the case of $\Delta \tau \neq 0$, the parameter configuration for blast-wave model can successfully describe the experimental results of collision energy and transverse momentum dependence of $R_{\text {side }}, R_{\text {out }}$, and $R_{\text {long. }}$. Since the collision system has different temperature at each centre-ofmass energy point, the configured parameters can be considered as the preferred values with a case of $T_{\text {kin }}$ as a function of $\sqrt{s_{\mathrm{NN}}}$ and $\Delta \tau \neq 0$ as shown in Figures 5 and 6 . However, it cannot be configured for the blast-wave parameter to fit the experimental data while setting $\Delta \tau$ to zero. This may imply that the particle emission duration plays an important role to describe the system expanding and cannot be ignored while calculating the $R_{\text {side }}, R_{\text {out }}$, and $R_{\text {long }}$ to fit the data at the same time. And the difference of $R_{\text {out }}^{2}-R_{\text {side }}^{2}$ presents a nonmonotonic increasing trend with the increasing of $\sqrt{s_{\mathrm{NN}}}$ as seen in the experimental analysis [20], which is sensitive to the equation of state and might be related to the critical end point with other observables taken into account.

\section{Competing Interests}

The authors declare that they have no competing interests.

\section{Acknowledgments}

This work was supported in part by the Major State Basic Research Development Program in China under Contract no. 2014CB845400, the National Natural Science Foundation of China under Contract nos. 11421505, 11220101005, 11105207, 11275250,11322547 , and U1232206, and the CAS Project Grant no. QYZDJ-SSW-SLH002.

\section{References}

[1] F. Karsch, "Lattice results on QCD thermodynamics," Nuclear Physics A, vol. 698, no. 1-4, pp. 199-208, 2002.

[2] I. Arsene, I. G. Bearden, D. Beavis et al., "Quark-gluon plasma and color glass condensate at RHIC? The perspective from the BRAHMS experiment," Nuclear Physics A, vol. 757, no. 1-2, pp. $1-27,2005$.

[3] B. B. Back, M. D. Baker, M. Ballintijn et al., "The PHOBOS perspective on discoveries at RHIC," Nuclear Physics A, vol. 757, no. 1-2, pp. 28-101, 2005. 
[4] J. Adames, M. M. Aggarwal, Z. Ahammed et al., "Experimental and theoretical challenges in the search for the quark-gluon plasma: the STAR Collaboration's critical assessment of the evidence from RHIC collisions," Nuclear Physics A, vol. 757, no. 1-2, pp. 102-183, 2005.

[5] K. Adcox, S. S. Adler, S. Afanasiev et al., "Formation of dense partonic matter in relativistic nucleus-nucleus collisions at RHIC: experimental evaluation by the PHENIX Collaboration," Nuclear Physics A, vol. 757, no. 1-2, pp. 184-283, 2005.

[6] J. Adams, M. M. Aggarwal, Z. Ahammed et al., "Azimuthal anisotropy and correlations at large transverse momenta in $p+p$ and $\mathrm{Au}+\mathrm{Au}$ Collisions at $\sqrt{s_{N N}}=200 \mathrm{GeV}$," Physical Review Letters, vol. 93, no. 25, Article ID 252301, 2004.

[7] J. Adams, M. M. Aggarwal, Z. Ahammed et al., "Multistrange baryon elliptic flow in Au+Au collisions at $\sqrt{s_{\mathrm{NN}}}=200 \mathrm{GeV}$," Physical Review Letters, vol. 95, no. 12, Article ID 122301, 6 pages, 2005.

[8] J. Adams, C. Adler, M. M. Aggarwal et al., “ $\phi$ meson production in $\mathrm{Au}+\mathrm{Au}$ and $\mathrm{p}+\mathrm{p}$ collisions at $\sqrt{s_{N N}}=200 \mathrm{GeV}$," Physics Letters B, vol. 612, no. 3-4, pp. 181-189, 2005.

[9] S. S. Adler, S. Afanasiev, C. Aidala et al., " $J / \psi$ production and nuclear effects for $d+\mathrm{Au}$ and $p+p$ Collisions at $\sqrt{s_{N N}}=$ 200 GeV," Physical Review Letters, vol. 96, no. 1, Article ID 012304, 2006.

[10] L. Adamczyk, G. Agakishiev, M. M. Aggarwal et al., "Inclusive charged hadron elliptic flow in $\mathrm{Au}+\mathrm{Au}$ collisions at $\sqrt{s_{N N}}=$ 7.7-39 GeV," Physical Review C, vol. 86, no. 5, Article ID 054908, 2012.

[11] K. Aamodt, B. Abelev, A. Abrahantes Quintana et al., "Chargedparticle multiplicity density at midrapidity in central $\mathrm{Pb}-\mathrm{Pb}$ collisions at $\sqrt{s_{N N}}=2.76 \mathrm{TeV}$," Physical Review Letters, vol. 105, no. 25, Article ID 252301, 11 pages, 2010.

[12] K. Aamodt, A. Abrahantes Quintana, D. Adamová et al., "Centrality dependence of the charged-particle multiplicity density at midrapidity in $\mathrm{Pb}-\mathrm{Pb}$ Collisions at $\sqrt{s_{N N}}=2.76 \mathrm{TeV}$," Physical Review Letters, vol. 106, no. 3, Article ID 032301, 2011.

[13] K. Aamodt, A. Abrahantes Quintana, D. Adamová et al., "Suppression of charged particle production at large transverse momentum in central $\mathrm{Pb}-\mathrm{Pb}$ collisions at $\sqrt{s_{N N}}=2.76 \mathrm{TeV}$," Physics Letters B, vol. 696, no. 1-2, pp. 30-39, 2011.

[14] B. Abelev, J. Adam, D. Adamová et al., "Centrality dependence of $\pi, K$, and $p$ production in $\mathrm{Pb}-\mathrm{Pb}$ collisions at $\sqrt{s_{N N}}=$ 2.76 TeV," Physical Review C, vol. 88, no. 4, Article ID 044910, 23 pages, 2013.

[15] N. Itoch, "Hydrostatic equilibrium of hypothetical quark stars," Progress of Theoretical Physics, vol. 44, no. 1, pp. 291-292, 1970.

[16] M. Alford, K. Rajagopal, and F. Wilczek, "QCD at finite baryon density: nucleon droplets and color superconductivity," Physics Letters, Section B: Nuclear, Elementary Particle and High-Energy Physics, vol. 422, no. 1-4, pp. 247-256, 1998.

[17] P. Costa, M. C. Ruivo, and C. A. de Sousa, “Thermodynamics and critical behavior in the Nambu-Jona-Lasinio model of QCD," Physical Review D, vol. 77, no. 9, Article ID 096001, 2008.

[18] Y. Lu, Y.-L. Du, Z.-F. Cui, and H.-S. Zong, "Critical behaviors near the (tri-)critical end point of QCD within the NJL model," The European Physical Journal C, vol. 75, article 495, 2015.

[19] R. A. Lacey, "Indications for a critical end point in the phase diagram for hot and dense nuclear matter," Physical Review Letters, vol. 114, no. 14, Article ID 142301, 5 pages, 2015.

[20] L. Adamczyk, J. K. Adkins, G. Agakishiev et al., "Beamenergy-dependent two-pion interferometry and the freeze-out eccentricity of pions measured in heavy ion collisions at the STAR detector," Physical Review C, vol. 92, no. 1, Article ID 014904, 2015.

[21] R. H. Brown and R. Q. Twiss, "A test of a new type of stellar interferometer on sirius," Nature, vol. 178, pp. 1046-1048, 1956.

[22] G. Goldhaber, S. Goldhaber, W. Lee, and A. Pais, "Influence of Bose-Einstein statistics on the antiproton-proton annihilation process," Physical Review, vol. 120, no. 1, pp. 300-312, 1960.

[23] S. E. Koonin, "Proton pictures of high-energy nuclear collisions," Physics Letters B, vol. 70, no. 1, pp. 43-47, 1977.

[24] S. Pratt, "Pion interferometry for exploding sources," Physical Review Letters, vol. 53, no. 13, pp. 1219-1221, 1984.

[25] D. H. Boal, C.-K. Gelbke, and B. K. Jennings, "Intensity interferometry in subatomic physics," Reviews of Modern Physics, vol. 62, no. 3, pp. 553-602, 1990.

[26] U. Heinz and B. V. Jacak, "Two-particle correlations in relativistic heavy-ion collisions," Annual Review of Nuclear and Particle Science, vol. 49, no. 1, pp. 529-579, 1999.

[27] M. A. Lisa, S. Pratt, R. Soltz, and U. Wiedemann, "Femtoscopy in relativistic heavy ion collisions: two decades of progress," Annual Review of Nuclear and Particle Science, vol. 55, no. 1, pp. 357-402, 2005.

[28] Y. B. Wei, Y. G. Ma, W. Q. Shen et al., "Exploring binding energy and separation energy dependences of HBT strength," Physics Letters B, vol. 586, no. 3-4, pp. 225-231, 2004.

[29] Y. G. Ma, Y. B. Wei, W. Q. Shen et al., "Surveying the nucleonnucleon momentum correlation function in the framework of quantum molecular dynamics model," Physical Review C, vol. 73, Article ID 014604, 2006.

[30] Z. Q. Zhang, S. Zhang, and Y. G. Ma, "Simulation of energy scan of pion interferometry in central $\mathrm{Au}+\mathrm{Au}$ collisions at relativistic energies," Chinese Physics $C$, vol. 38, no. 1, Article ID 014102, 2014.

[31] J. Yang and W.-N. Zhang, "Interferometry analyses of pion and kaon for the granular sources for $\mathrm{Au}+\mathrm{Au}$ collisions at $\sqrt{s_{N N}}=$ 200 GeV," Nuclear Science and Techniques, vol. 27, no. 6, article $147,2016$.

[32] L. Adamczyk, J. K. Adkins, G. Agakishiev et al., "Observation of charge asymmetry dependence of pion elliptic flow and the possible chiral magnetic wave in heavy-ion collisions," Physical Review Letters, vol. 114, no. 25, Article ID 252302, 2015.

[33] J. Adams, M. M. Aggarwal, Z. Ahammed et al., "Proton- $\Lambda$ correlations in central $\mathrm{Au}+\mathrm{Au}$ collisions at $\sqrt{s_{N N}}=200 \mathrm{GeV}$," Physical Review C, vol. 74, Article ID 064906, 2006.

[34] L. Adamczyk, J. K. Adkins, G. Agakishiev et al., "Measurement of interaction between antiprotons," Nature, vol. 527, pp. 345348, 2015.

[35] J. Adams, M. M. Aggarwal, Z. Ahammed et al., "Pion interferometry in $\mathrm{Au}+\mathrm{Au}$ collisions at $\sqrt{s_{N N}}=200 \mathrm{GeV}$," Physical Review C, vol. 71, no. 4, Article ID 044906, 2005.

[36] B. I. Abelev, M. M. Aggarwal, Z. Ahammed et al., "Pion interferometry in $\mathrm{Au}+\mathrm{Au}$ and $\mathrm{Cu}+\mathrm{Cu}$ collisions at $\sqrt{s_{N N}}=62.4$ and $200 \mathrm{GeV}$," Physical Review C, vol. 80, Article ID 024905, 2009.

[37] S. S. Adler, S. Afanasiev, C. Aidala et al., "Bose-einstein correlations of charged pion pairs in $\mathrm{Au}+\mathrm{Au}$ Collisions at $\sqrt{s_{N N}}=$ 200 GeV," Physical Review Letters, vol. 93, no. 15, Article ID 152302, 2004.

[38] A. Adare, S. Afanasiev, C. Aidala et al., "Systematic study of charged-pion and kaon femtoscopy in $\mathrm{Au}+\mathrm{Au}$ collisions at $\sqrt{s_{N N}}=200 \mathrm{GeV}$,' Physical Review C, vol. 92, no. 3, Article ID 034914, 2015. 
[39] K. Aamodt, A. Abrahantes Quintana, D. Adamová et al., “Twopion Bose-Einstein correlations in central $\mathrm{Pb}-\mathrm{Pb}$ collisions at $\sqrt{\mathcal{s}_{\mathrm{NN}}}=2.76$ TeV," Physics Letters B, vol. 696, no. 4, pp. 328-337, 2011.

[40] A. Adare, S. Afanasiev, C. Aidala et al., "Beam-energy and system-size dependence of the space-time extent of the pion emission source produced in heavy ion collisions," https://arxiv .org/abs/1410.2559.

[41] B. I. Abelev, M. M. Aggarwal, Z. Ahammed et al., "Systematic measurements of identified particle spectra in $p p, d+\mathrm{Au}$, and $\mathrm{Au}+\mathrm{Au}$ collisions at the STAR detector," Physical Review C, vol. 79, no. 3, Article ID 034909, 58 pages, 2009.

[42] L. Kumar, "Systematics of kinetic freeze-out properties in high energy collisions from STAR," Nuclear Physics A, vol. 931, pp. 1114-1119, 2014.

[43] J. Adams, C. Adler, M. M. Aggarwal et al., "Identified particle distributions in $p p$ and $\mathrm{Au}+\mathrm{Au}$ Collisions at $\sqrt{s_{N N}}=200 \mathrm{GeV}$," Physical Review Letters, vol. 92, no. 11, Article ID 112301, 2004.

[44] F. Retière and M. A. Lisa, "Observable implications of geometrical and dynamical aspects of freeze-out in heavy ion collisions," Physical Review C-Nuclear Physics, vol. 70, no. 4, Article ID 044907, 2004.

[45] S. Zhang, L. X. Han, Y. G. Ma, J. H. Chen, and C. Zhong, "Production and ratio of $\pi, K, p$, and $\Lambda$ in $\mathrm{Pb}+\mathrm{Pb}$ collisions at $\sqrt{s_{N N}}=2.76 \mathrm{TeV}$," Physical Review $C$, vol. 89, no. 3, Article ID 034918, 2014.

[46] L. Xue, Y. G. Ma, J. H. Chen, and S. Zhang, "Production of light (anti)nuclei, (anti)hypertriton, and di- $\Lambda$ in central $\mathrm{Au}+\mathrm{Au}$ collisions at energies available at the BNL Relativistic Heavy Ion Collider," Physical Review C, vol. 85, no. 6, Article ID 064912, 2012.

[47] N. Shah, Y. G. Ma, J. H. Chen, and S. Zhang, "Production of multistrange hadrons, light nuclei and hypertriton in central $\mathrm{Au}+\mathrm{Au}$ collisions at $\sqrt{s_{\mathrm{NN}}}=11.5$ and $200 \mathrm{GeV}$," Physics Letters $B$, vol. 754, pp. 6-10, 2016.

[48] B. Tomášik, "DRAGON: Monte Carlo generator of particle production from a fragmented fireball in ultrarelativistic nuclear collisions," Computer Physics Communications, vol. 180, no. 9, pp. 1642-1653, 2009.

[49] M. Chojnacki, A. Kisiel, W. Florkowski, and W. Broniowski, "THERMINATOR 2: THERMal heavy IoN generATOR 2," Computer Physics Communications, vol. 183, no. 3, pp. 746-773, 2012.

[50] A. Kisiel, T. Tałuć, W. Broniowski, and W. Florkowski, “THERMINATOR: THERMal heavy-IoN generATOR," Computer Physics Communications, vol. 174, no. 8, pp. 669-687, 2006.

[51] U. A. Wiedemann, "Two-particle interferometry for noncentral heavy-ion collisions," Physical Review C, vol. 57, no. 1, pp. 266279, 1998.

[52] S. Pratt, T. Csörgő, and J. Zimányi, "Detailed predictions for two-pion correlations in ultrarelativistic heavy-ion collisions," Physical Review C, vol. 42, no. 6, pp. 2646-2652, 1990.

[53] G. Bertsch, M. Gong, and M. Tohyama, "Pion interferometry in ultrarelativistic heavy-ion collisions," Physical Review C, vol. 37, no. 5, pp. 1896-1900, 1988. 

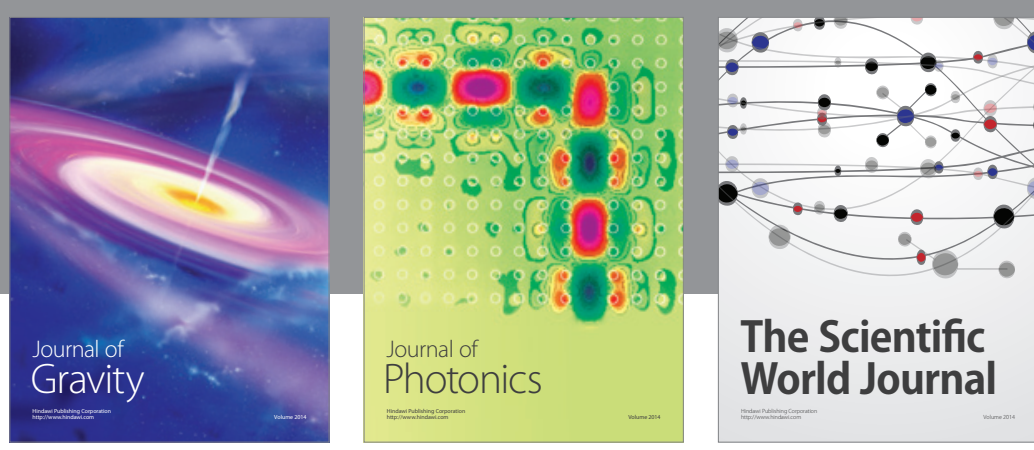

The Scientific World Journal
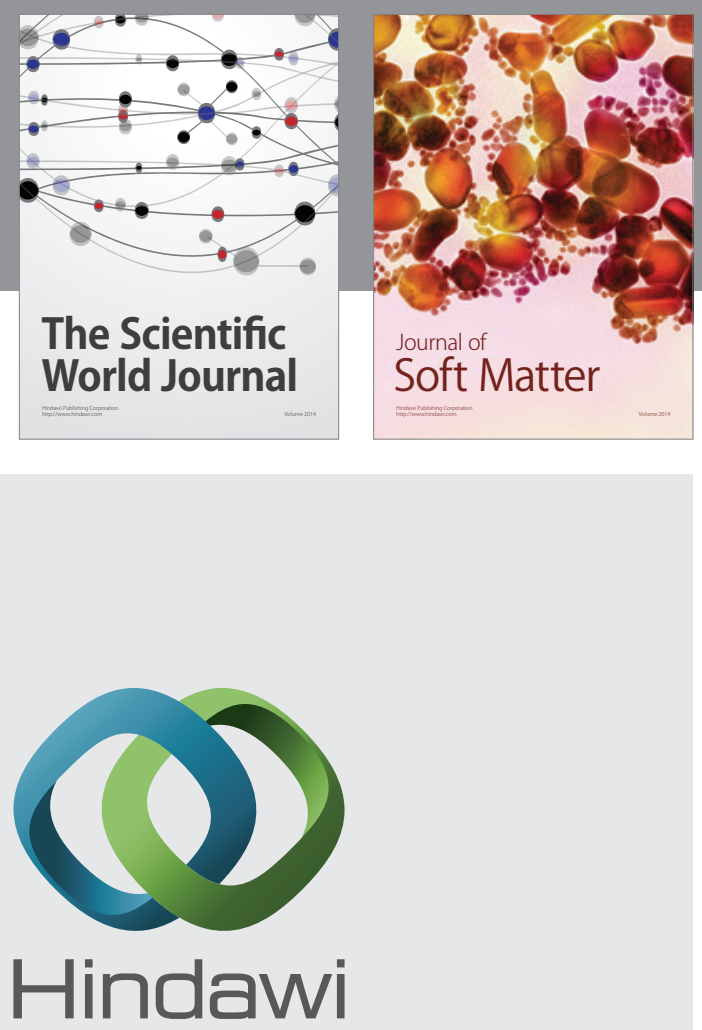

Submit your manuscripts at

http://www.hindawi.com

nternational Journal of

Statistical Mechanics
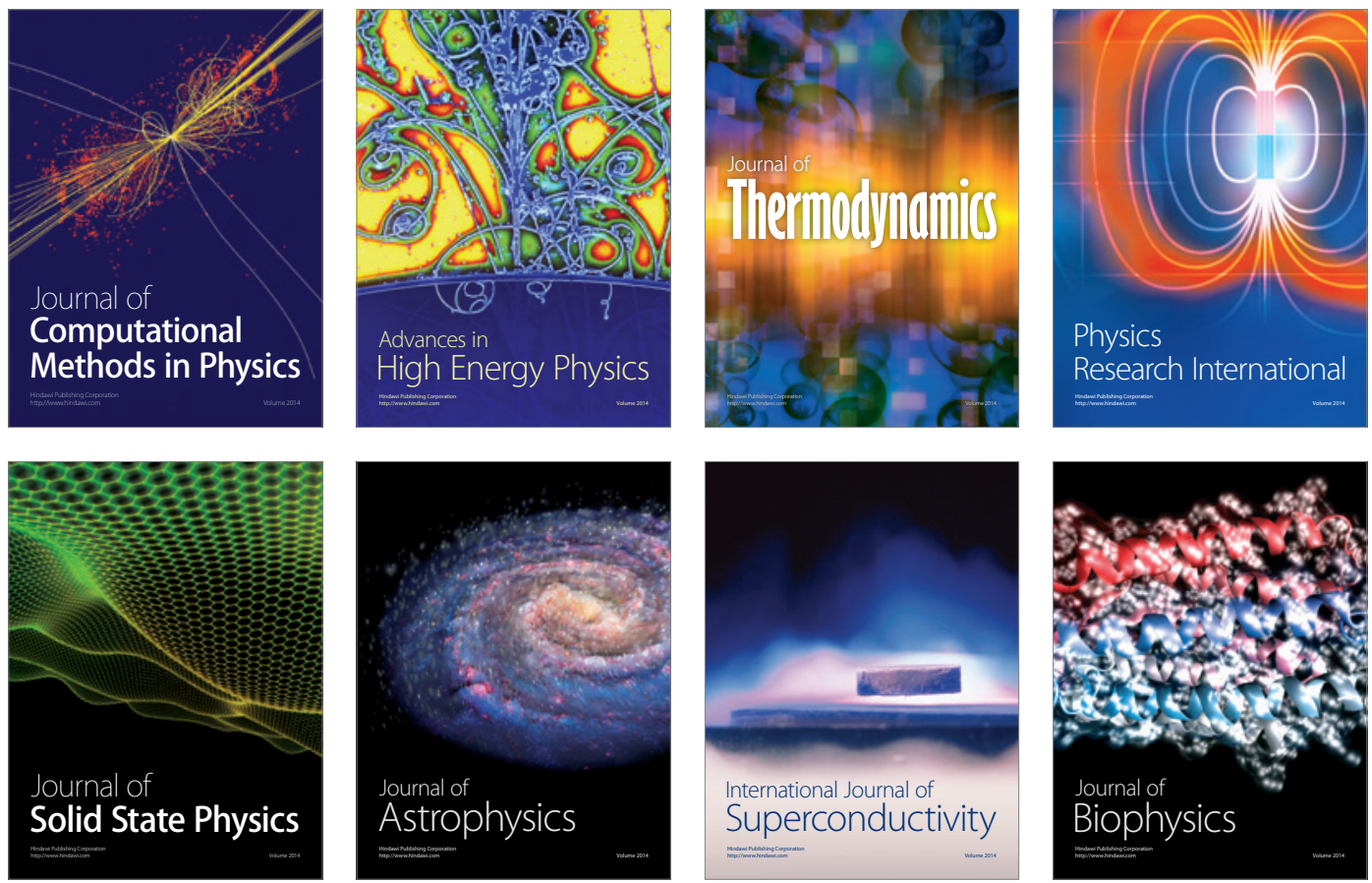
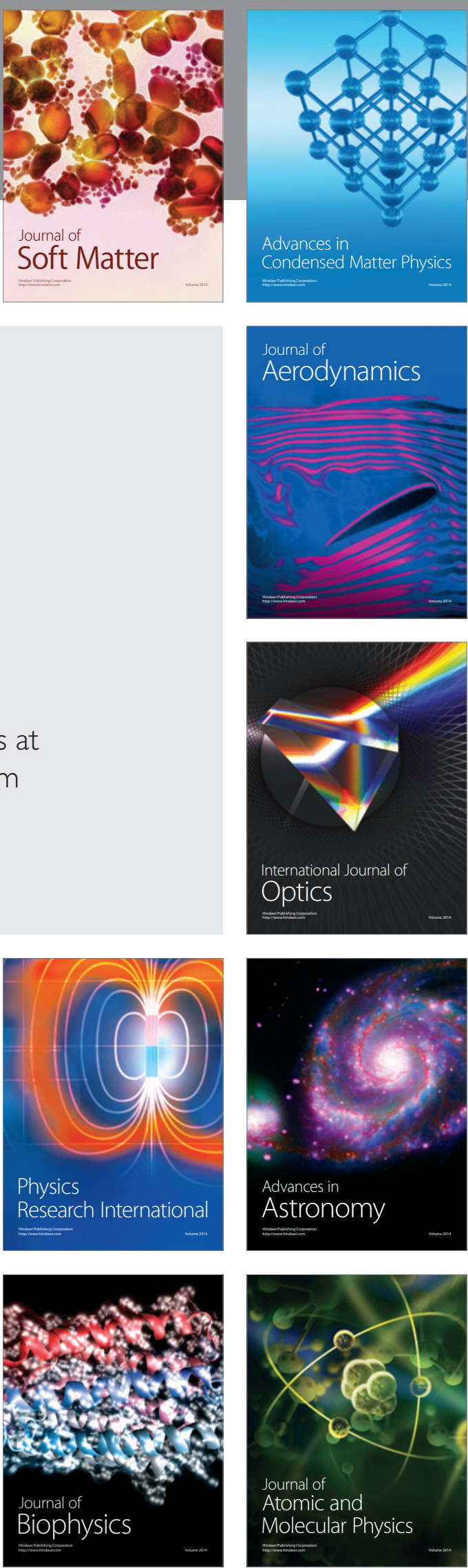